\title{
Ovarian serous cystadenofibroma: Towards a preoperative diagnosis
}

\section{Ekanayake CD ${ }^{1}$, Dissanayake $D^{2}$, Kumarasinghe $\mathbf{I}^{3}$}

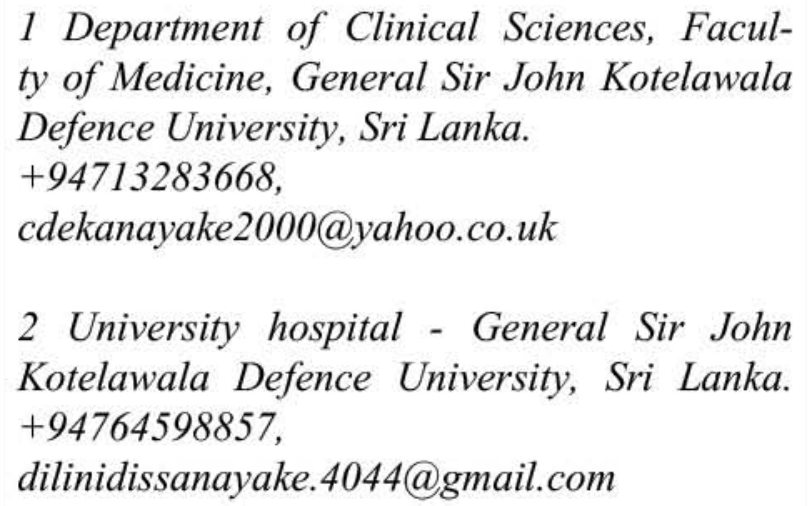

1 Department of Clinical Sciences, Faculty of Medicine, General Sir John Kotelawala Defence University, Sri Lanka.

+94713283668 , cdekanayake2000@yahoo.co.uk

2 University hospital - General Sir John Kotelawala Defence University, Sri Lanka. +94764598857,

dilinidissanayake.4044@gmail.com

3 Department of Para Clinical Sciences, Faculty of Medicine, General Sir John Kotelawala Defence University, Sri Lanka.

+94777703351,iranthiihs@yahoo.com

Corresponding Author-Dr. CD Ekanayake Email: cdekanayake2000@yahoo.co.uk

\section{Abstract}

\section{Background:}

Ovarian cystadenofibromas are rare benign ovarian neoplasms which contains epithelial and fibrous stromal components which give rise to complex cystic and solid areas which will be visualized sonographically as malignant tumors. The management for cystadenofibroma of ovary is complete surgical removal of the tumour. The prognosis is generally excellent with prompt and appropriate treatment as there is a very low risk of recurrence.

\section{Case presentation:}

A 45-year-old woman presented to the gynaecology clinic complaining of irregular men- strual bleeding, secondary dysmenorrhea and left sided lower abdominal pain of one month duration. On bimanual examination a cystic, nontender, left adnexal mass was found. Imaging revealed a complex cystic mass in left ovary $(10 \times 9.6 \times 8 \mathrm{~cm})$. There was a thin film of pelvic free fluid adjacent to the inferior margin of the lesion. Few non-specific subcentrimetric para aortic, left iliac lymph nodes were seen. CA-125 level was $179 \mathrm{U} / \mathrm{ml}$.

A staging laparotomy was performed and included total abdominal hysterectomy, bilateral salphyngoophorectomy and infracolic omentectomy. Bilateral multilocular ovarian cysts (left cyst $-10 \times 8 \mathrm{~cm}$ and the right cyst $-3 \times$ $4 \mathrm{~cm}$ ) were present. Histology revealed bilateral serous cystadenofibromas. No atypical or malignant cells were observed in peritoneal washings.

\section{Conclusion:}

Ovarian cystadenofibromas appear malignant on imaging and macroscopy. Frozen sections does not appear to be a reliable indicator and only MRI appears to be dependable in predicting the diagnosis of a cystadenofibroma prior to histological confirmation.

\section{Keywords:}

serous cystadenofibroma, complex cystic ovarian mass, ovarian mass, ovarian cyst

\section{Background}

Ovarian cystadenofibromas are rare benign ovarian neoplasms containing epithelial and fibrous 
stromal components which give rise to complex cystic and solid areas. These are visualized as malignant tumours on imaging ${ }^{1,2}$. These neoplasms present with abdominal pain, vaginal bleeding or a palpable mass in the abdomen but most are asymptomatic and are detected incidentally. Presence of the complications due to these ovarian tumours are rare, but may include rupture of the cystic portion or torsion of the affected ovary ${ }^{2}$.

The management of serous cystadenofibroma of ovary is surgical excision. The prognosis is generally excellent with prompt and appropriate treatment. They have a very low risk of recurrence on compete removal through surgery ${ }^{2}$.

\section{Case presentation}

A 45-year-old Sinhalese woman with no fertility wishes, presented to the gynaecology clinic of the University Hospital - General Sir John Kotelawala Defense University, complaining of irregular menstrual bleeding, secondary dysmenorrhea and left sided lower abdominal pain of one month duration. She had no loss of appetite or loss of weight. She was a known diabetic and was on metformin. She had undergone two benign breast lump excisions in 2013 and 2015. There was no family history of malignancy.

On examination, she was afebrile and had a soft abdomen. Her cervix appeared normal. Bimanual examination revealed an anteverted uterus of normal size and a cystic non tender left adnexal mass. Imaging (pelvic ultrasound and computerised tomography) revealed a complex cystic mass in left adenexa of ovarian origin $(10 \times 9.6 \times 8 \mathrm{~cm}$ in size). A thin film of free fluid was adjacent to the inferior margin of the lesion. Few non-specific subcentrimetric para aortic and left iliac lymph nodes were seen. CA-125 level was $179 \mathrm{U} / \mathrm{ml}$.
A staging laparotomy inclusive of a total abdominal hysterectomy, bilateral salphingo-ophorectomy and infracolic omentectomy was performed. Peritoneal washings were also obtained. Bilateral multilocular ovarian cysts with solid areas (left cyst $-10 \times 8 \mathrm{~cm}$ and the right cyst $-3 \times 4 \mathrm{~cm}$ ) were found (Fig. 1). The cysts were removed intact without any spillage. There were no palpable lymph nodes or deposits in the paracolic gutters. Liver surface and under surface of the diaphragm were also normal. The patient made an uneventful postoperative recovery and was discharged home on the third postoperative day. Histology revealed bilateral serous cystadenofibromas (Fig. 2). No atypical or malignant cells were observed in peritoneal washings.

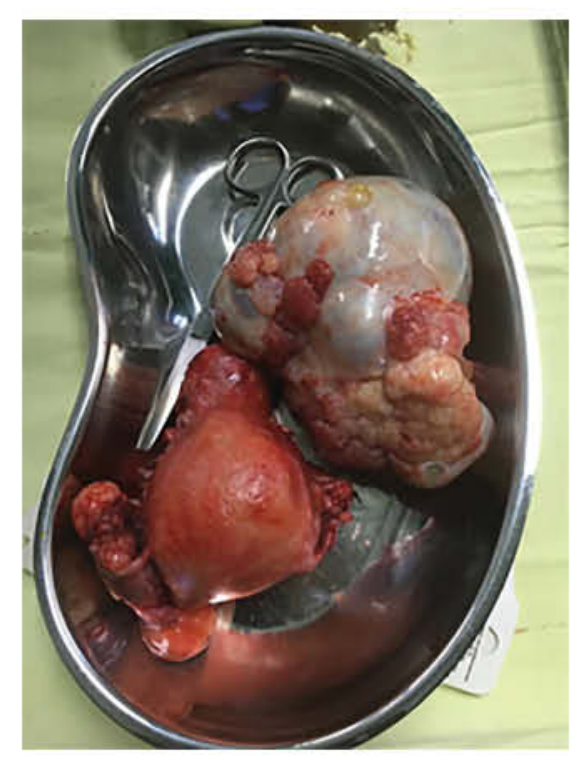

Fig. 1. Macroscopic appearance

\section{Discussion}

This case illustrates the difficulty of diagnosing a cystadenofibroma prior to surgery because they appear malignant on imaging and macroscopy. The preoperative differential diagnosis for this patient included an early-stage epithelial ovarian cancer based on imaging and CA-125 level. The CA-125 can be marginally elevated in cystadenofibromas. This is thought to be due to peritoneal irritation ${ }^{3}$. 


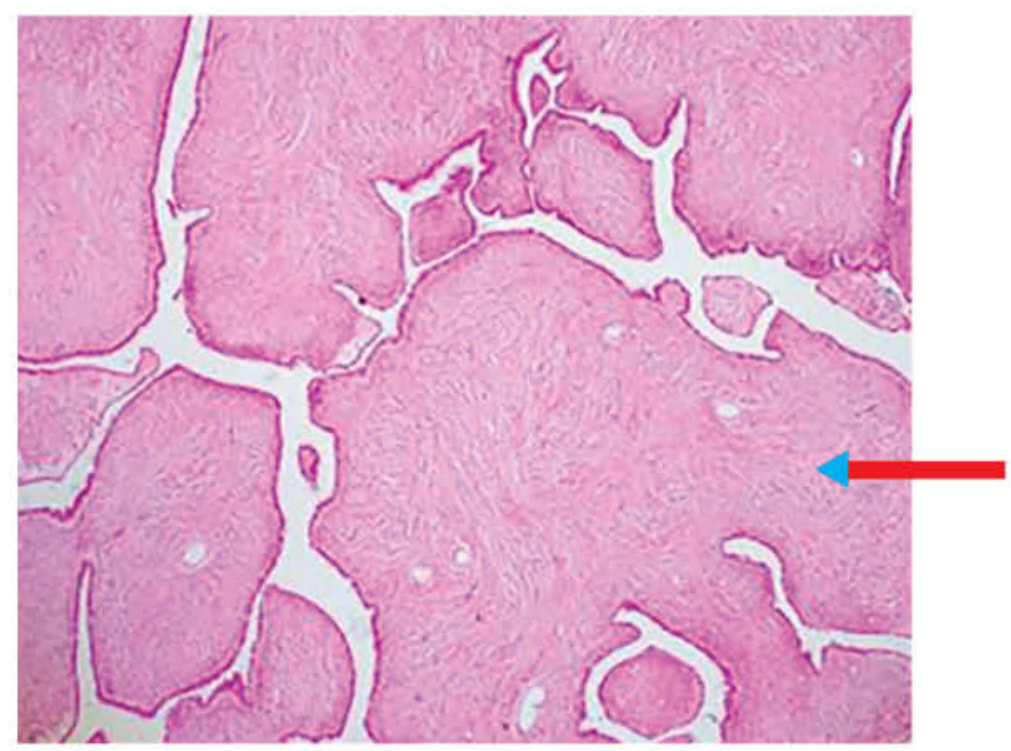

Simple papillary processes with a predominatly fibrous stroma

\section{X10}

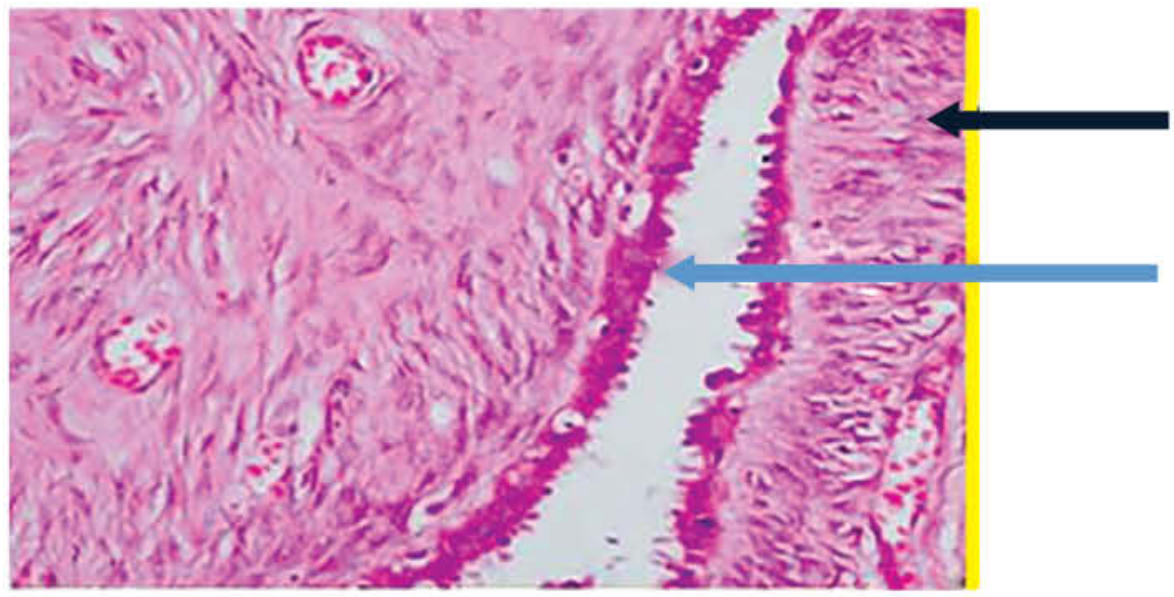

Fibrous stroma

Serous epithelial lining

\section{40}

Fig 02. Microscopic appearance of serous cystadenofibroma

Both ultrasound scanning and computed tomography are of limited value in the preoperative diagnosis of cystadenofibromas as they are seen to have either a solitary or multilocular cyst with papillary projections and a variable amount of solid components ${ }^{1,4}$. This heterogenous appearance makes its differentiation from a malignant neoplasm difficult. However, magnetic resonance imaging (MRI) is considered to be more accurate in terms of diagnosing cystadenofibromas. The characteristic fibrous stroma causes a very low signal intensity on T2 weighted images and is considered the key MRI feature in the diagnosis of tumours with fibrous element such as cystadenofibroma and brenner's tumour ${ }^{1}$.

Macroscopically, cystadenofibromas comprise solid and cystic areas with papillary projections on the surface as well as within the cystic cavity. This appearance makes it impossible to differentiate them from malignant and borderline tumours during surgery. There is some evidence to support 
the use of frozen sections which are thought to help in avoiding extensive surgery ${ }^{5}$. However, the sensitivity of frozen sections is $100 \%$ for benign looking tumours whereas for malignant tumours it is $96 \%{ }^{6}$. Thus, if frozen sections are done there is a theoretical risk of doing suboptimal excision in $4 \%$ of malignancies. This risk of missing one in twenty five malignancies is likely to be an unacceptable error rate considering prognosis and subsequent therapeutic implications.

\section{Conclusion}

Ovarian cystadenofibromas are rare benign ovarian neoplasms which appear malignant on imaging and macroscopy. Frozen sections does not appear to be a reliable indicator and only MRI appears to be dependable in predicting the diagnosis of a cystadenofibroma prior to histological confirmation.

\section{$\underline{\text { Acknowledgements }}$}

We express our sincere gratitude to the members of the obstetrics and gynaecology ward, University Hospital - Kotelawala Defence University, Werahera, Sri Lanka.

\section{$\underline{\text { References }}$}

1. Wasnik A, Elsayes K. Ovarian cystadenofibroma. A masquerader of malignancy. Indian Journal of Radiology and Imaging 2010; 20(4):297-299.

2. Leelavathi RP, Sree SG, Srirama S. Ovarian Serous Cystadenofibroma : A Rare Case Report. Indian Journal of Obstetrics and Gynaecology Research 2015; 2(4):297-299

3. Christine J, Tolmanl, Tejasvini V, Henk WR. Schreuder1 Extremely elevated CA-125 in benign ovarian disease due to stretch of the peritoneum. BMJ Case Rep. 2012; Available at: https://www.ncbi.nlm.nih.gov/pmc/articles/PMC4543764/

4. Topçu HO, Oskovi ZA, Güzel AI, Özer I, Kokanali MK. An Incidental Diagnosis of the Cystadenofibroma of the Ovary in the Cesarean Section: A Case Report. Obstet Gynecol Cases Rev, 2014; 1:002

5. Cho DH. Serous cystadenofibroma misdiagnosed as an ovarian malignancy. BMJ Case Rep 2018; 11:e228223.

6. Hashmi AA, Naz S, Edhi MM, et al. Accuracy of intraoperative frozen section for the evaluation of ovarian neoplasms: an institutional experience. World J Surg Onc 2016; 14: 91. Available at: https://doi.org/10.1186/ s12957-016-0849-x 


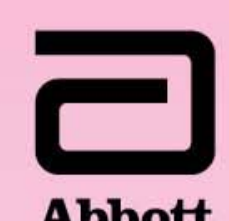

Abbott

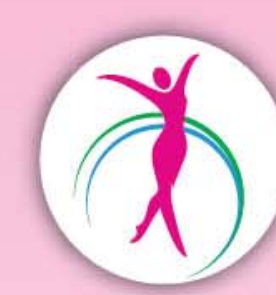

\section{IMPROVES THE MENOPAUSAL SYMPTOMS ${ }^{1-3}$}

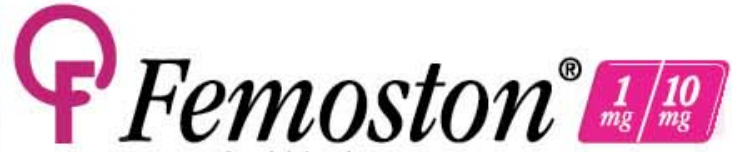 estradiol/dydrogesterone}

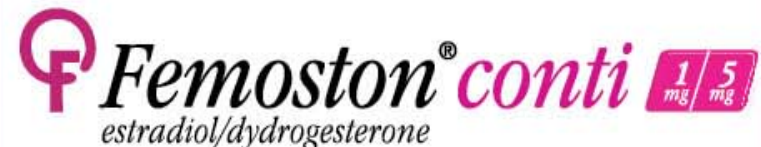

\section{ధFemoston 2 [2] estradiol/dydrogesterone}

\section{Fem}

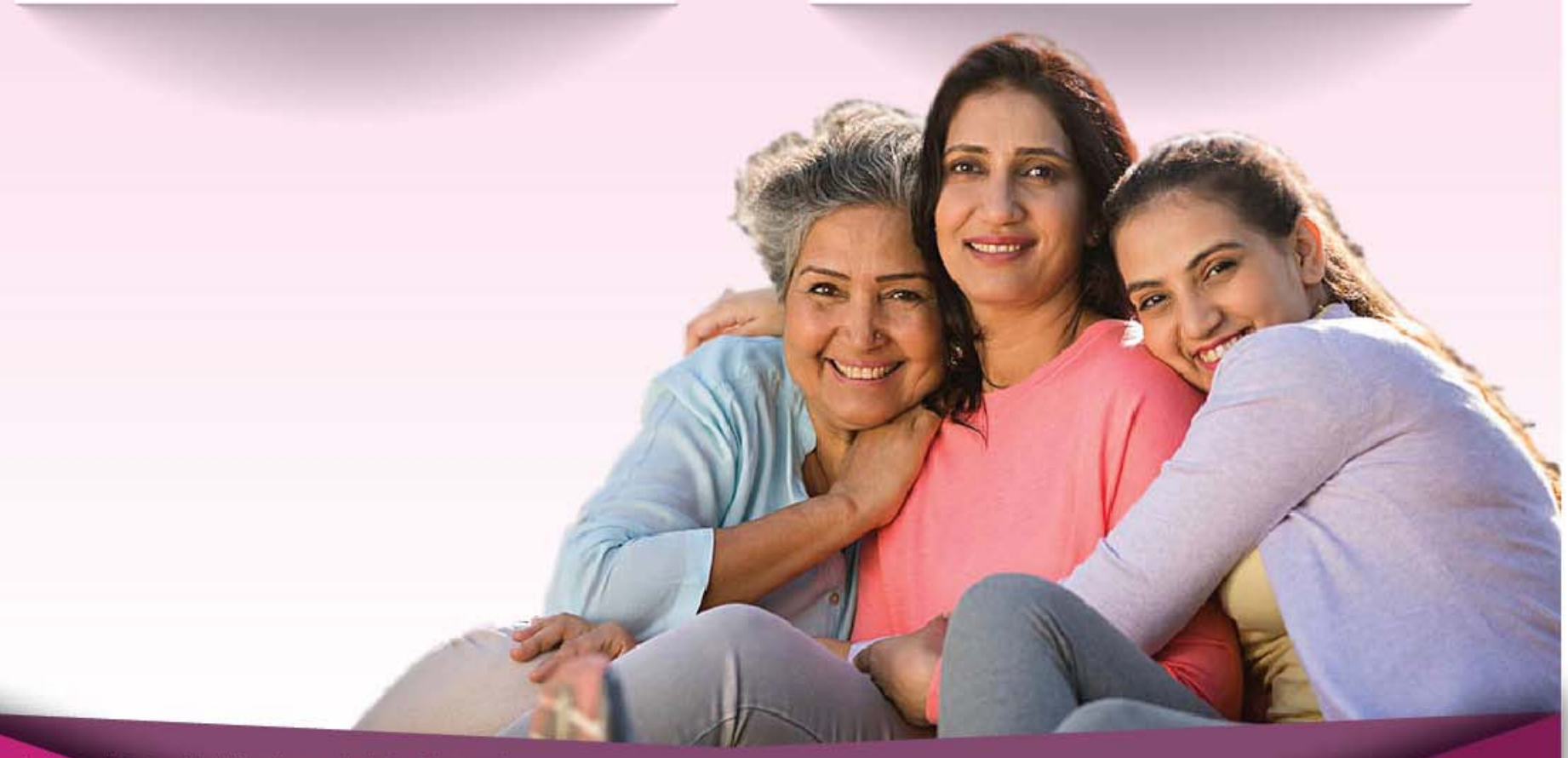

For the use of a Regi stered Medical Pracitioner or a Hospital or a Laboratory only.

Image for representallion purpose only, not of actual patient

Q Registered Trademark of the Abbott Group of Companies.

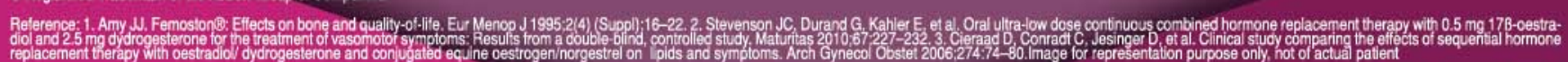

A

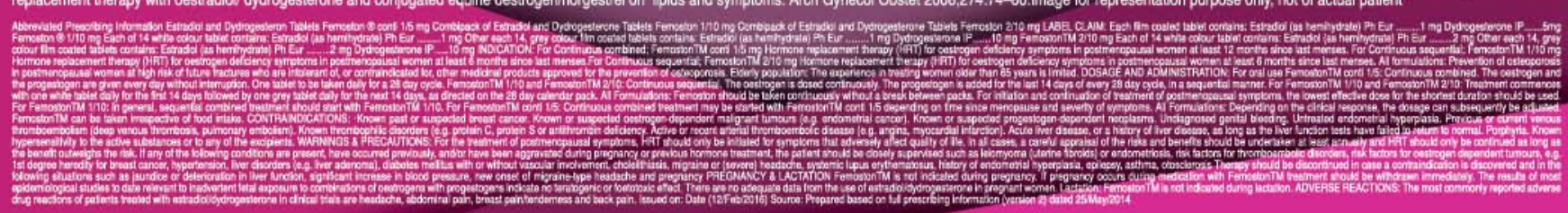

\section{CIC $1=1$}

No. 112, Model Farm Road, Colombo 8, Sri Lanka. 